\title{
Uso de medicamentos asociados al riesgo de caídas en ancianos no institucionalizados
}

\author{
Risk of drug-related falls among noninstitutionalized older adults \\ Uso de medicamentos associados ao risco de quedas em idosos não institucionalizados
}

\author{
Begoña Pellicer Garcíaํㅜ, Sergio Moreno González ${ }^{1}$, Antonio Manuel Cardoso Muñoz ${ }^{2}$, Isabel Antón-Solanas ${ }^{1}$, Vicente \\ Gea Caballero ${ }^{3}$, Raúl Juárez Vela ${ }^{1}$
}

Cómo citar este artículo:

Pellicer García B, Moreno González S, Cardoso Muñoz AM, Antón-Solanas I, Gea Caballero V, Juárez Vela R. Risk of drug-related falls among noninstitutionalized older adults. Rev Esc Enferm USP. 2018;52:e03319. DOI: http://dx.doi.org/10.1590/S1980-220X2017012603319

${ }^{1}$ Universidad San Jorge de Zaragoza, Facultad de Ciencias de la Salud, Zaragoza, España.

${ }^{2}$ Universidad de Salamanca, Departamento de Enfermería y Fisioterapia, Salamanca, España.

${ }^{3}$ Universidad de Valencia, Escuela

Enfermería La Fe, Valencia, España.

\begin{abstract}
Objective: To determine the number of drugs taken per day, which represents a risk factor for falls among noninstitutionalized older adults with a history of falls in the last year. Method: This was a descriptive study that used random sampling and the following measurement instruments: the WHO questionnaire for the study of falls in older adults, gait scale and geriatric depression scale and gait and balance. Univariate and bivariate analysis, nonparametric chi-squared test, and binary logistic regression were performed using the SPSS statistical program version 21.0. Results: 214 individuals participated in the study. Those who took $\geq 4$ drugs presented higher risk of falling, $\mathrm{p}=0.010 \mathrm{OR}=4.034$. The same was not true for individuals who took $\leq 3$ drugs, $p=0.006$ $\mathrm{OR}=0.335$. Conclusion: The use of four or more drugs per day was considered a risk factor for falls among older adults.
\end{abstract}

\section{DESCRIPTORS}

Aged; Accidental Falls; Drug Utilization; Gait; Postural Balance; Geriatric Nursing. 


\section{INTRODUCCIÓN}

El envejecimiento poblacional es un fenómeno evidente a nivel mundial y hasta el momento los medicamentos son la herramienta terapéutica más empleada para aliviar los síntomas de enfermedades, mejorar el estado funcional y la calidad de vida en la población anciana ${ }^{(1)}$.

Un elemento sustancial en la determinación del riesgo de caídas en ancianos es el uso de medicamentos, ya que un elevado consumo actúa como un factor de riesgo para futuras caídas ${ }^{(2)}$. Aproximadamente $25 \%-38 \%$ de las personas ancianas que viven en la comunidad se caen al menos una vez al año ${ }^{(3-4)}$, este hecho genera en la persona numerosas repercusiones a nivel físico (siendo las fractura la consecuencia física más seria), psicológico (miedo a nuevas caídas, ansiedad, depresión) y social (aislamiento en las relaciones sociales) ${ }^{(5)}$, conllevando un aumento de la fragilidad y discapacidad ${ }^{(6)}$. La prevención y promoción de la salud son herramientas importantes para reducir la aparición de estos eventos y minimizar las complicaciones secundarias derivadas de las caídas ${ }^{(7-8)}$.

El término polifarmacología no tiene una definición establecida como tal, y algunos autores la definen como el uso concomitante de cinco o más medicamentos ${ }^{(9-10)}$. Está demostrado que a mayor número de medicamentos prescritos la posibilidad de que aparezcan efectos adversos se incrementa sustancialmente, pero no se ha determinar un número exacto ${ }^{(11-12)}$. La literatura científica expone que un consumo de igual o mayor a cuatro medicamentos diarios se considera un factor de riesgo de caídas en poblaciones ancianas ${ }^{(13)}$.

Estudios encontrados examinan la incidencia de caídas en personas ancianas que toman determinados tipos de medicamentos, pero son muy pocos los autores que correlacionan el número de medicamentos con el riesgo de caídas, y mucho menos cuando se trata de poblaciones con antecedentes de caída ${ }^{(14-15)}$. El objetivo de este estudio es determinar y establecer qué número de medicamentos consumidos diariamente es influyente en el riesgo de caídas en ancianos no institucionalizados y con historial de caídas en el último año.

\section{MÉTODO}

\section{DiseÑo DEL ESTUDio}

Se realizó un estudio descriptivo transversal en dos centros sociales de Zaragoza (España), desde mayo a julio de 2015. Para la obtención de la muestra se utilizó un muestreo de tipo aleatorio.

Los Centros de Convivencia para Mayores están vinculados al ayuntamiento de la ciudad de Zaragoza, siendo espacios de encuentro y relación destinados a ofrecer a las personas mayores no institucionalizadas servicios y actividades adecuados a sus necesidades e intereses. Constituyen una red de equipamientos y servicios para la participación, el encuentro, la convivencia y el ocio. La red de centros oferta numerosas actividades adecuadas a los intereses de los mayores, el soporte para el desarrollo personal y social, para la participación y el compromiso.

\section{Medida de la muestra y TIPO De MuestreO}

El tamaño muestral se calculó mediante la fórmula de estimación de la proporción con una población en estudio de (N) 472 personas $\geq$ a 65 años, un nivel de confianza (1- $\alpha)$ del $95 \%$, una precisión (d) $\pm 0,5$ y heterogeneidad del $50 \%$. De este modo se obtuvo un tamaño muestral de (n) 213 personas $\geq$ a 65 años. Se incluyeron todas aquellas personas que cumplían los criterios de inclusión establecidos y todas aquellas personas que mostraron deseo de participar.

\section{CRITERIOS DE SELECCIÓN}

Se incluyeron en el estudio personas $\geq$ a 65 años, que hubieran sufrido al menos una caída en el último año previa al inicio de este estudio, usuarios de los dos centros seleccionados, no institucionalizados, sin deterioro cognitivo (siendo valorado mediante el Pfeiffer Short Portable Mental Questionnaire (PSMSQ) en su versión española) ${ }^{(16)}$ y con aceptación del consentimiento informado.

\section{VARIABLES E INSTRUMENTOS DE MEDIDA}

Variables sociodemográficas: sexo, edad, estado civil y vivir solo.

\section{OTRAS VARIABLES E INSTRUMENTOS DE MEDIDA}

Cuestionario de la Organización Mundial de la Salud (OMS) para el estudio de caídas en el anciano ${ }^{(17)}$. Evalúa los aspectos relacionados con la historia de la caída. Este cuestionario no otorga una puntuación numérica. Para este estudio se recogieron las siguientes variables: práctica de ejercicio siendo valorada en aquellas personas que al menos tuviesen una dedicación mínima de 1 hora semanal, el uso de ayudas para la deambulación, la incontinencia urinaria, el miedo a caer, número de caídas en el último año (1 caída $o \geq 2$ caídas). La variable dependiente el número de medicamentos consumidos diariamente ( $\leq$ a 3 medicamentos/ día e $\geq$ a 4 medicamentos/día).

El riesgo de caída se evaluó mediante la escala de marcha y equilibrio de Tinetti ${ }^{(18)}$. Evalúa el riesgo de caída, la máxima puntuación corresponde a 28 puntos, 12 puntos para la marcha y 16 puntos para el equilibrio. La suma de ambas puntuaciones otorga la puntuación de riesgo o no de caída en una persona. A mayor puntuación menor riesgo, puntuaciones totales $\leq$ a 24 puntos indican riesgo de caídas.

La depresión se evaluó con la escala Depresión Geriátrica de Yesavage en su versión española ${ }^{(19)}$. Evalúa la presencia de riesgo de depresión en población geriátrica. La puntuación total corresponde a 15 puntos, una puntuación de 0 a 4 puntos indica ausencia de depresión mientras que puntuaciones de 5 a 15 informa de la existencia de depresión.

\section{RECOGIDA DE DATOS}

Todos los participantes fueron entrevistados tras una cita telefónicamente programada. A cada participante se le realizó una entrevista individual semiestructurada en su centro de referencia. 


\section{ANÁLISIS ESTADÍSTICO}

La codificación, el procesamiento de los datos y el análisis estadístico se realizó con el programa estadístico SPSS versión 21. Los principales estimadores se presentaron con un intervalo de confianza del 95\%, margen de error del 5\% y una significación estadística de $\mathrm{p}=<0,05$. Se realizó un análisis estadístico univariante y bivariante con prueba no paramétrica de Chi-cuadrado $\left(\mathrm{X}^{2}\right)$. Análisis de regresión logística bivariante donde se analizaron aquellas variables independientes con asociaciones significativas al número de medicamentos consumidos diariamente ( $\leq$ a 3 medicamentos e $\geq$ a 4 medicamentos).

\section{Cuestiones ÉTICAs}

Previamente al comienzo del estudio, el Comité de Ética de Investigación Clínica de Aragón (CEICA) informó favorablemente la solicitud del proyecto. Todos los participantes ofrecieron su consentimiento informado por escrito al inicio de la entrevista.

\section{RESULTADOS}

La muestra se correspondió con un total de 213 personas (hombres y mujeres), las características sociodemográficas de la muestra se incluyen en la tabla 1 . Un 79,3\% de los participantes eran mujeres y un 20,7\% hombres, siendo la media de edad de 78 años (rango de 65 a 97 años) con $\mathrm{DE} \pm 6,999$. E1 95\% de los participantes consumía al menos un medicamento diario, siendo la media de medicamentos de 5,38 con una $\mathrm{DE} \pm$ 3,501 y cuyos límites fueron de 0 a 17 medicamentos por día.

En el análisis bivariado el 65,3\% consumían $\geq$ a 4 medicamentos diarios, mientras que un $34,7 \%$ consumía $\leq$ a 3 ) encontrándose un mayor número de consumo de medicamentos en edades comprendidas entre los 76 y 86 años. La prevalencia de riesgo de caída fue del 31,9\% sobre el total de la muestra, para el grupo de personas que consumía $\geq$ a 4 medicamentos fue del $41,7 \%$, frente al 13,5\% para el grupo que consumía $\leq$ a 3. La mitad de las personas que consumían $\geq$ a 4 medicamentos diarios eran viudas, mientras que para el grupo que consumía $\leq$ a 3 eran casados, también una de cada tres personas que consumían $\geq$ a 4 medicamentos usaban ayudas técnicas para la deambulación. E1 67\% de las personas que vivían solas también consumían $\geq$ a 4 medicamentos frente al 33\% de los que vivían solos y consumían $\leq$ a 3 . La incontinencia urinaria también tuvo una mayor prevalencia en las personas que consumían $\geq$ a 4 medicamentos siendo del $69,7 \%$ y del 30,3\% para los de $\leq$ a 3 . Existe un elevado número de personas que realizan actividad física, pero conforme la muestra se divide por número de medicamentos se observa que las prevalencias se mantienen.

Tres de cada cuatro personas sufren más de una caída al año independientemente del número de medicamentos consumidos. También se observa que el miedo a sufrir nuevas caídas está muy presente en este tipo de población, independientemente del número de medicamentos consumidos. Sobre la población general, aproximadamente un tercio presenta depresión, conforme se analiza según el número de medicamentos consumidos se obtiene que un $78,3 \%$ de los que tienen depresión consumían $\geq$ a 4 medicamentos frente a un $21,7 \%$ que consumía $\leq$ a 3 medicamentos.

Con prueba no paramétrica de $\mathrm{X}^{2}$ y un IC del 95\% se analizaron todas la variables independientes frente a las variables dependientes (consumo $\geq$ a 4 medicamentos y los que tomaban $\leq$ a 3 medicamentos). Se obtuvieron asociaciones estadísticamente significativas para las variables edad, uso de ayudas para la deambulación, actividad física, riesgo de caída y depresión en el grupo de consumo $\geq$ a 4 medicamentos (Tabla 1). Mientras que en el grupo de $\leq$ a 3 fueron la incontinencia urinaria $\mathrm{p}=0,041$, la actividad física $\mathrm{p}=0,029$ y el riesgo de caída $\mathrm{p}=0,001$.

Tabla 1 - Análisis univariante, bivariante y prueba X² para consumo $\geq$ a 4 medicamentos diarios - Zaragoza (España), mayo - julio de 2015.

\begin{tabular}{|c|c|c|c|c|}
\hline VARIABLES & $\begin{array}{c}\leq 3 \\
\text { Medicamentos } \\
\mathrm{n}=74(34,7 \%) \\
\end{array}$ & $\begin{array}{c}\geq 4 \\
\text { Medicamentos } \\
n=139(65,3 \%)\end{array}$ & $\begin{array}{c}\text { TOTAL } \\
\mathrm{n}=213(\mathbf{1 0 0} \%)\end{array}$ & $\underset{(<0,05)}{p}$ \\
\hline \multicolumn{5}{|l|}{ Sexo } \\
\hline Hombres & $14(18,9)$ & $30(21,6)$ & $44(20,7)$ & \multirow[t]{2}{*}{0,647} \\
\hline Mujeres & $60(81,1)$ & $109(78,4)$ & $169(79,3)$ & \\
\hline \multicolumn{5}{|l|}{ Edad } \\
\hline [de 65 a 75] & $41(55,4)$ & $52(37,5)$ & $93(43,7)$ & \multirow{3}{*}{0,041} \\
\hline [de 76 a 86] & $27(36,5)$ & $75(53,9)$ & $102(47,9)$ & \\
\hline [de 87 a 97] & $6(8,1)$ & $12(8,6)$ & $18(8,4)$ & \\
\hline \multicolumn{5}{|l|}{ Estado civil } \\
\hline Soltero & $6(8,1)$ & $10(7,2)$ & $16(7,5)$ & \multirow[t]{5}{*}{0,402} \\
\hline Casado & $32(43,2)$ & $52(37,4)$ & $84(39,4)$ & \\
\hline Viudo & $29(39,2)$ & $71(51,1)$ & $100(46,9)$ & \\
\hline Separado & $1(1,4)$ & $1(0,7)$ & $2(0,9)$ & \\
\hline Divorciado & $6(8,1)$ & $5(3,6)$ & $11(5,1)$ & \\
\hline \multicolumn{5}{|c|}{ Ayudas para deambular } \\
\hline Sí & $10(13,5)$ & $40(28,8)$ & $50(23,5)$ & \multirow[t]{2}{*}{0,012} \\
\hline No & $64(86,5)$ & $99(71,2)$ & $163(76,5)$ & \\
\hline \multicolumn{5}{|l|}{ Vive solo } \\
\hline Sí & $31(41,9)$ & $63(45,3)$ & $94(44,1)$ & \multirow[t]{2}{*}{0,631} \\
\hline No & $43(58,1)$ & $76(54,7)$ & $119(55,9)$ & \\
\hline
\end{tabular}




\begin{tabular}{|c|c|c|c|c|}
\hline \multicolumn{5}{|c|}{ Incontinencia urinaria } \\
\hline Sí & $27(36,5)$ & $62(44,6)$ & $89(41,8)$ & \multirow[t]{2}{*}{0,253} \\
\hline No & $47(63,5)$ & $77(55,4)$ & $124(58,2)$ & \\
\hline \multicolumn{5}{|l|}{ Actividad física } \\
\hline Sí & $59(79,7)$ & $81(58,3)$ & $140(65,7)$ & \multirow[t]{2}{*}{0,002} \\
\hline No & $15(20,3)$ & $58(41,7)$ & $73(34,2)$ & \\
\hline \multicolumn{5}{|c|}{ Caídas en el último año } \\
\hline 1 caída & $19(25,7)$ & $35(25,2)$ & $54(25,3)$ & \multirow[t]{2}{*}{0,937} \\
\hline$\geq$ a 2 caídas & $55(74,3)$ & $104(74,8)$ & $159(74,6)$ & \\
\hline \multicolumn{5}{|l|}{ Miedo a caer } \\
\hline Sí & $47(63,5)$ & $82(59,0)$ & $129(60,5)$ & \multirow[t]{2}{*}{0,520} \\
\hline No & $27(36,5)$ & $57(41,0)$ & $84(39,4)$ & \\
\hline \multicolumn{5}{|c|}{ Riesgo de caída/Tinetti } \\
\hline$=<24^{\mathrm{a}}$ & $10(13,5)$ & $58(41,7)$ & $68(31,9)$ & \multirow[t]{2}{*}{$<0,001$} \\
\hline$=>25 \mathrm{~b}^{\mathrm{b}}$ & $64(86,5)$ & $81(58,3)$ & $145(68,0)$ & \\
\hline$=>5^{\mathrm{c}}$ & $13(17,6)$ & $47(33,8)$ & $60(28,1)$ & \multirow[t]{2}{*}{0,012} \\
\hline$=<4^{\mathrm{d}}$ & $61(82,4)$ & $92(66,2)$ & $153(71,8)$ & \\
\hline
\end{tabular}

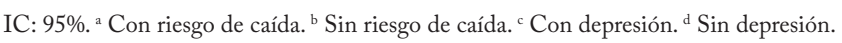

En el análisis de regresión logística binaria se introdujeron todas las variables independientes asociadas al consumo $\geq$ a 4 medicamentos y se obtuvo que el consumo $\geq$ a 4 medicamentos se comportan para el riesgo de caída en poblaciones ancianas no institucionalizadas y con historia previa de caída en los últimos 12 meses $p=0,010 \mathrm{OR}=4,034$ (Tabla 2). Esto mismo sucede de manera inversa para personas con un consumo $\leq$ a 3 medicamentos $\mathrm{p}=0,006 \mathrm{OR}=0,335$, siendo $\mathrm{OR}<1$ que actuaría como un factor protector (Tabla 3). Es aquí, donde se observa que un consumo a partir de 4 medicamentos es un factor de riesgo para personas que están en riesgo de caída. Aquella persona cuyo perfil sea el de una mujer de 84 años, con un consumo $\geq$ a 4 medicamentos diarios, viuda, que viva sola, no use ayudas técnicas para la deambulación, con incontinencia urinaria, que no realice actividad física, que tenga miedo y haya sufrido más de una caída en el último año y depresión presentará un 99,5\% de probabilidad a sufrir nuevas caídas.

Tabla 2 - Análisis de regresión logística binaria de todas las variables con asociación estadísticamente significativa en prueba X², para un consumo $\geq$ a 4 fármacos diarios - Zaragoza (España), mayo - julio de 2015.

\begin{tabular}{|c|c|c|c|c|c|}
\hline & \multirow{2}{*}{ B } & \multirow{2}{*}{ Sig. } & \multirow{2}{*}{$\operatorname{Exp}(B)$} & \multicolumn{2}{|c|}{ I.C. $95 \%$ para EXP(B) } \\
\hline & & & & Inf. & Sup. \\
\hline Edad & , 110 & ,697 & 1,116 & ,642 & 1,940 \\
\hline Ayudas para deambular &,- 347 &, 533 & ,707 & ,237 & 2,104 \\
\hline Actividad física &,- 694 & ,056 & ,499 & ,245 & 1,017 \\
\hline Depresión &, 155 & ,710 & 1,168 &, 515 & 2,648 \\
\hline Riesgo de caída & 1,395 & , 010 & 4,034 & 1,393 & 11,681 \\
\hline Constante &, 610 & ,268 & 1,841 & ----- & ----- \\
\hline
\end{tabular}

Exp(B): Odds ratio. Sig.: Nivel de significación. IC: Intervalo de confianza.

Tabla 3 - Análisis de regresión logística binaria de todas las variables con asociación estadísticamente significativa en prueba X², para un consumo $\leq$ a 3 fármacos diarios - Zaragoza (España), mayo - julio de 2015.

\begin{tabular}{|c|c|c|c|c|c|}
\hline & \multirow{2}{*}{ B } & \multirow{2}{*}{ Sig. } & \multirow{2}{*}{$\operatorname{Exp}(B)$} & \multicolumn{2}{|c|}{ I.C. $95 \%$ para EXP(B) } \\
\hline & & & & Inf. & Sup. \\
\hline Actividad física &, 347 &, 341 & 1,415 & ,693 & 2,890 \\
\hline Riesgo de caída & $-1,093$ & ,006 &, 335 & , 153 & ,734 \\
\hline Incontinencia urinaria &,- 535 & , 101 &, 586 & ,309 & 1,111 \\
\hline Constante &,- 595 & , 104 & ,552 & ----- & ----- \\
\hline
\end{tabular}

Exp(B): Odds ratio. Sig.: Nivel de significación. IC: Intervalo de confianza.

\section{DISCUSIÓN}

La mayoría de los estudios encontrados en la literatura científica analizan la incidencia de caídas en personas que toman determinados tipos de medicamentos, pero por el momento hay muy pocos estudios que correlacionen el número de medicamentos consumidos con el riesgo de caídas y menos aún, cuando se trata de poblaciones con historial de caídas en el último año ${ }^{(14-15)}$. Consideramos que este tipo poblacional, está expuesto a un mayor riesgo de experimentar nuevas caídas, por lo que se les puede considerar 
como candidatos ideales para examinar la influencia que tiene el número de medicamentos consumidos diariamente. Como se ha visto en otros estudios, un consumo elevado de medicamentos incrementa el riesgo de caídas, pudiendo resultar mucho más peligroso que la clase de medicamento que toman en cuestión ${ }^{(20-21)}$. También establecen que cuanto mayor es el número de medicamentos consumidos, la probabilidad de caer es mucho mayor. Se han encontrado asociaciones significativas entre la depresión y el riesgo de caída ${ }^{(20)}$, aunque en nuestro estudio no se han encontrado dichas asociaciones. Este hecho puede ser debido a que la población estudiada no se encontraba institucionalizada.

Un hallazgo singular de nuestro estudio, es la significación estadística encontrada entre el riesgo de caídas y tomar z a 4 medicamentos, considerándose un factor de riesgo en este tipo de población, mientras que para un consumo $\leq$ a 3 medicamentos no fue encontrada dicha significación. Nuestro estudio es consistente y los resultados son semejantes con un estudio publicado en el año 2012 aunque hay que destacar que sus resultados tuvieron una significación menor respecto a los resultados de nuestro estudio ( $\mathrm{p}=0,027$ OR:1,14 $\mathrm{n}=116)$. Esta diferencia puede ser debida a diferencias en el tamaño muestral y en la recogida de datos, ya que la obtención de los datos se realizó mediante revisión de historias clínicas ${ }^{(2)}$. Otro estudio encontrado también analizó la asociación entre el número de medicamentos consumidos y el riesgo de caída, aunque éstos obtuvieron asociaciones significativas a partir de un consumo $\geq$ a 5 medicamentos diarios, independientemente de la tipología del medicamento consumido ${ }^{(22)}$. Un estudio publicado y realizado en personas hospitalizadas, cuyo objetivo era conocer la importancia de las caídas asociadas a la medicación informaron datos próximos a los de nuestro estudio en cuanto a que una de cada tres personas sufre una caída de tipo recurrente anualmente. En ese estudio un 77,1\% de la población analizada tomaba 5 o más medicamentos, encontrando una media de 7,4 medicamentos consumidos diariamente, siendo la media superior respecto a la encontrada en nuestro estudio. Sin embargo, la muestra que estudiaron se compuso de personas de todo tipo de edades. De las 214 personas analizadas, el 88,8\% de la muestra tenían más de 60 años (190 personas). La variable edad fue un factor influyente en el riesgo de caída, ya que observaron que cuanto mayor era la edad existía una mayor vulnerabilidad a sufrir futuras caídas. Este estudio obtuvo asociaciones significativas entre el hecho de tener caídas recurrentes y un mayor uso de medicamentos relacionados con el riesgo de caídas ( $\mathrm{p}=0,0001)$. En nuestro estudio se obtuvieron asociaciones significativas a partir de un consumo $\geq \mathrm{a} 4$ medicamentos consumidos diariamente ${ }^{(23)}$. Nuestro estudio constó de población válida no institucionalizada y todos ellos mayores de 65 años, aunque en nuestros resultados la variable edad no se asoció al riesgo de caída, la única variable encontrada fue el consumo $\geq$ a 4 medicamentos diarios.

Consideramos que nuestro estudio, aporta hallazgos interesantes y clínicamente relevantes afirmando que un consumo $\geq$ a 4 medicamentos es un factor de riesgo de caídas. Estos hallazgos deben servir de recordatorio a los profesionales sanitarios y principalmente a los del ámbito de la atención primaria, para sopesar los beneficios de la farmacoterapia frente al riesgo potencial de caídas en personas susceptibles. La gran mayoría de los estudios encontrados investigan asociaciones entre el número y tipo de medicamentos consumidos con las caídas.

Este estudio considera que se debe investigar los factores de riesgo antes de que se produzca la caída como tal. Es decir, nuevos estudios cuyas investigaciones vayan enfocadas a la detección del riesgo de caídas y que permitan analizar los posibles factores de riesgo que puedan estar asociados. Todo esto nos permitirá mejorar los protocolos sobre prevención y actuaciones ante las caídas. El riesgo de caídas debe ser evaluado con mayor frecuencia puesto que es un importante detector de la fragilidad, esto nos permitirá evitar o prevenir en la medida de lo posible futuras caídas que puedan presentar este tipo de población e incluso es posible conseguir atrasar la dependencia y discapacidad, por ello es importante seguir explorando este fenómeno.

\section{LIMITACIONES}

Este estudio presenta como limitación la imposibilidad de conocer la adherencia al tratamiento farmacológico.

\section{CONCLUSIÓN}

Un consumo a partir de cuatro medicamentos diarios se considera un factor de riesgo muy influyente para todas aquellas personas ancianas no institucionalizadas que hayan sufrido al menos una caída en el último año y se encuentren en riesgo de caídas.

\section{RESUMEN}

Objetivo: Determinar qué número de medicamentos consumidos diariamente es influyente en el riesgo de caídas en ancianos no institucionalizados y con historial de caídas en el último año. Método: Estudio descriptivo mediante muestreo aleatorio con la utilización de los siguientes instrumentos de medida: cuestionario de la OMS para el estudio de caídas en el anciano, escala de marcha y escala depresión geriátrica y escala de marcha y equilibrio. El análisis univariante, bivariante con prueba no paramétrica de Chi-cuadrado y regresión logística binaria se ellevó a cabo con el programa estadístico SPSS versión 21.0. Resultados: Participaron del estudio 213 personas. El consumo $\geq$ a 4 medicamentos se comportan para el riesgo de caída $p=0,010$ OR=4,034. Esto mismo no sucede para personas con un consumo $\leq$ a 3 medicamentos $\mathrm{p}=0,006 \mathrm{OR}=0,335$. Conclusión: Un consumo a partir de cuatro o más medicamentos diarios se considera un factor de riesgo para las personas ancianas que se encuentren en riesgo de caídas.

\section{DESCRIPTORES}

Anciano; Accidentes por Caídas; Utilización de Medicamentos; Marcha; Balance Postural; Enfermería Geriátrica.

\section{RESUMO}

Objetivo: Determinar o número de medicamentos consumidos diariamente que influencia o risco de quedas em idosos não institucionalizados e com histórico de quedas no último ano. Método: Estudo descritivo mediante amostragem aleatória com a utilização dos seguintes instrumentos 
de medida: questionário da OMS para o estudo de quedas no idoso, escala de marcha, escala de depressão geriátrica e escala de marcha e equilíbrio. A análise univariante, bivariante, com prova não paramétrica de qui-quadrado e regressão logística binária foi realizada com o programa estatístico SPSS versão 21.0. Resultados: Participaram do estudo 213 pessoas. O consumo $\geq$ a 4 medicamentos comporta-se como um fator de risco de queda $\mathrm{p}=0,010 \mathrm{OR}=4,034$. $\mathrm{O}$ mesmo não acontece para pessoas com um consumo $\leq$ a 3 medicamentos $\mathrm{p}=0,006 \mathrm{OR}=0,335$. Conclusão: Um consumo a partir de quatro medicamentos diários é considerado um fator de risco para as pessoas idosas que se encontrem em risco de quedas.

\section{DESCRITORES}

Idoso; Acidentes por Quedas; Uso de Medicamentos; Marcha; Equilíbrio Postural; Enfermagem Geriátrica.

\section{REFERENCIAS}

1. Regueiro M, Mendy N, Cañás M, Farina HO, Nagel P. Uso de medicamentos en adultos mayores no institucionalizados. Rev Peru Med Exp Salud Publica. 2011;28(4):643-7.

2. Freeland KN, Thompson AN, Zhao Y, Leal JE, Mauldin PD, Moran WP. Medication use and associated risk of falling in a geriatric outpatient population. Ann Pharmacother. 2012;46(9):1188-92. DOI: http://dx.doi.org/10.1345/aph.1q689

3. Swift CG, Iliffe S. Assessment and prevention of falls in older people: concise guidance. Clin Med (Lond). 2014;14(6):658-62. DOI: http://dx.doi.org/10.7861/clinmedicine.14-6-658

4. Fhon JR, Rosset I, Freitas CP, Silva AO, Santos JL, Rodrigues RA. Prevalence of falls among frail elderly adults. Rev Saúde Pública. 2013;47(2):266-73. DOI: http://dx.doi.org/10.1590/S0034-8910.2013047003468

5. Salvá A, Bolíbar I, Pera G, Arias C. Incidence and consequences of falls among elderly people living in the community. Med Clin (Barc). 2004;122(5):172-6. DOI: http://dx.doi.org/10.1016/s0025-7753(04)74184-6

6. Gill TM, Murphy TE, Gahbauer EA, Allore HG. Association of injurious falls with disability outcomes and nursing home admissions in community-living older persons. Am J Epidemiol. 2013;178(3):418-25. DOI: http://dx.doi.org/10.1093/aje/kws554

7. Riera R, Trevisani VFM, Ribeiro JPN. Osteoporose: a importância da prevenção de quedas. Rev Bras Reumatol. 2003;43(6):364-8. DOI: http://dx.doi.org//10.1590/s0482-50042003000600008

8. Rubenstein LZ. Falls in older people: epidemiology, risk factors and strategies for prevention. Age Ageing. 2006;35 Suppl 2:ii37-41. DOI: http://dx.doi.org/10.1093/ageing/afl084

9. Linjakumpu T, Hartikainen S, Klaukka T, Veijola J, Kivelä SL, Isoaho R. Use of medications and polypharmacy are increasing among the elderly. J Clin Epidemiol. 2002;55(8):809-17. DOI: http://dx.doi.org/10.1016/s0895-4356(02)00411-0

10. Gallagher P, O'Mahony D. STOPP (Screening Tool of Older Persons' potencially inappropriate Prescriptions): application to acutely ill elderly patients and comparison with Beers' criteria. Age Ageing. 2008;37(6):673-9. DOI: http://dx.doi.org/10.1093/ageing/afn197

11. Chumney EC, Rovinson LC. The effects of pharmacist interventions on patients with polypharmacy. Pharm Pract (Granada). 2006;4(3):103-9. DOI: http://dx.doi.org/10.4321/s1885-642×2006000300001

12. Routledge PA, O'Mahony MS, Woodhouse KW. Adverse drug reactions in elderly patients. Br J Clin Pharmacol. 2003;57(2):121-6. DOI: http://dx.doi.org/10.1046/j.1365-2125.2003.01875.x

13. Weber V, White A, Mcllvried R. An electronic medical record (EMR) based intervention to reduce polypharmacy and falls in an ambulatory rural elderly population. J Gen Intern Med. 2008;23(4):399-404. DOI: http://dx.doi.org/10.1007/s11606-007-0482-z

14. Fletcher PC, Berg K, Dalby DM, Hirdes JP. Risk factors for falling among community-based seniors. J Patient Saf. 2009;5(2):61-6. DOI: http://dx.doi:10.1097/PTS.0b013e3181a551ed

15. Ganz DA, BaoY, Shekelle PG, Rubenstein LZ. Will my patient fall? JAMA. 2007;297(1):77-86. DOI: http://dx.doi.org/10.1001/jama.297.1.77

16. González-Montalvo JI, Rodríguez L, Ruipérez I. Validación del cuestionario de Pfeiffer y la escala de incapacidad mental de la Cruz Roja en la detección del deterioro mental en los pacientes externos de un servicio de geriatría. Rev Esp Geriatr Gerontol. 1992;27:129-33.

17. Vidán MT, Vellas B, Montemayor T, Romer C, Garry PJ, Ribera JM, et al. Cuestionario de la OMS para el estudio de las caídas en el anciano. Rev Esp Geriatr Gerontol. 1993;28(1):41-8.

18. Tinetti ME. Performance-oriented assessment of mobility problems in elderly patients. J Am Geriatr Soc. 1986;34(2):119-26.

19. Martínez J, Onís MC, Dueñas R, Albert C, Aguado C, Luque R. Versión española del cuestionario de Yesavage abreviado (GDS) para el despistaje de depresión en mayores de 65 años: adaptación y validación. Medifam. 2002;12(10):620-30. DOI: http://dx.doi.org/10.4321/s1131-57682002001000003

20. Deandrea S, Lucenteforte E, Bravi F, Foschi R, Vecchia CL, Negri E. Risk factors for falls in community-dwelling older people: a systematic review and meta-analysis. Epidemiology. 2010;21(5):658-68. DOI: http://dx.doi.org/10.1097/ede.0b013e3181e89905

21. Huang ES, Karter AJ, Danielson KK, Warton EM, Ahmed AT. The association between number of prescription medications and incident falls in a multi-ethnic population of adult type-2 diabetes patients: the diabetes and aging study. J Gen Intern Med. 2009;25(2):141-6. DOI: http://dx.doi.org/10.1007/s11606-009-1179-2

22. Kojima T, Akishita M, Nakamura T, Nomura K, Ogawa S, lijima K, et al. Polypharmacy as a risk for fall occurrence in geriatric outpatients. Geriatr Gerontol Int. 2012;12(3):425-30. DOI: http://dx.doi:10.1111/j.1447-0594.2011.00783

23. Costa-Dias MJ, Oliveira AS, Martins T, Araújo F, Santos AS, Moreira CN, et al. Medication fall risk in old hospitalized patients: a retrospective study. Nurse Educ Today. 2014;34(2):171-6. DOI: http://dx.doi.org/10.1016/j.nedt.2013.05.016 